\title{
The adult penile urethra is a novel entry site for HIV-1 that preferentially targets resident urethral macrophages
}

\author{
Y Ganor ${ }^{1,2,3}$, Z Zhou ${ }^{1,2,3}$, J Bodo ${ }^{1,2,3}$, D Tudor ${ }^{1,2,3}$, J Leibowitch ${ }^{4,5}$, D Mathez ${ }^{4}$ A Schmitt ${ }^{2,3,6}$, \\ M-C Vacher-Lavenu ${ }^{7}, \mathrm{M} \mathrm{Revol}^{8}$ and $\mathrm{M}$ Bomsel $^{1,2,3}$
}

The penile urethra is routinely targeted by sexually transmitted bacterial and viral pathogens, and also represents a probable site for HIV type-1 (HIV-1) entry. Yet, the mechanisms of urethral HIV-1 transmission are unknown. To describe the initial steps of penile HIV-1 entry, we obtained whole penile tissues from individuals undergoing elective gender reassignment and developed ex vivo polarized explants of different penile epithelia, as well as in vitro immunocompetent reconstructed urethra. In penile explants, $1 \mathrm{~h}$ exposure to cell-associated HIV-1 results in higher HIV-1 entry into the urethra, whereas the fossa navicularis and glans are relatively resistant to HIV-1. CCR5 + /CD4 + urethral macrophages are the initial cells infected by HIV-1, which exit the epithelial compartment following inoculation with cell-associated HIV-1 that induces decreased CCL2/MCP-1 production. Urethral T cells are mostly CD8 + or naive CD4 +, and not infected by HIV-1 on its early entry. In urethral reconstructions, efficient translocation of cell-associated HIV-1 depends on viral tropism (R5 $>$ X4) and can be decreased by gp41-specific IgAs. Cell-free HIV-1 is inefficient at urethral penetration. Our results identify the male urethra as a novel entry site for HIV-1 that targets resident urethral macrophages. These results might explain the incomplete prophylactic efficacy of male circumcision in reducing HIV-1 transmission.

\section{INTRODUCTION}

HIV type-1 (HIV-1) is mainly transmitted during sexual intercourse and, hence, needs to enter and translocate across mucosal surfaces. A detailed comprehension of HIV-1 transmission in the female genital and anorectal tracts was facilitated because of the availability of relevant mucosal tissues for research (for review see ref. 1). In sharp contrast, the limited accessibility of tissues originating from the male genital tract, and especially the adult penis, has resulted in a lack of information regarding the precise mechanism of penile HIV-1 transmission. HIV-1 infection in men has only recently regained extensive attention following reports of three clinical trials, which clearly demonstrated that male circumcision provides $50-60 \%$ protection from $\mathrm{HIV}-1$ acquisition, ${ }^{2-4}$ suggesting an important role of the male foreskin as an entry portal for HIV-1. Indeed, our previous studies described the cellular and molecular mechanisms responsible for HIV-1 entry into the inner foreskin. ${ }^{5-7}$ Yet, the protective outcome of circumcision is far from being complete, pointing to the existence of other penile epithelia as potential HIV-1 entry sites.

The penile urethra represents an additional probable site for HIV-1 entry, as it is lined only by a pseudostratified nonkeratinized columnar epithelium. In contrast, the more distal fossa navicularis region (termed herein the fossa) is a stratified non-keratinized epithelium and the outermost glans region is a stratified keratinized epithelium. ${ }^{8-11}$ Importantly, the urethra is the initial site of exposure to and infection by both bacterial and viral pathogens (e.g., N. gonorrhea, C. trachomatis, E. coli,

\footnotetext{
${ }^{1}$ Department of Mucosal Entry of HIV-1 and Mucosal Immunity, Cell Biology, and Host Pathogen Interactions, Cochin Institute, INSERM, U1016, Paris, France. ${ }^{2} \mathrm{CNRS}$, UMR 8104, Paris, France. ${ }^{3}$ Paris Descartes University, Sorbonne Paris Cité, Paris, France. ${ }^{4}$ Immunology and Virology Unit, Department of Internal Medicine and Infectious Diseases, Raymond Poincaré Hospital, Garches, France. ${ }^{5}$ University and Medical School, Saint Quentin en Yvelines, Versailles, France. ${ }^{6}$ Electron Microscopy Platform, Cochin Institute, INSERM, U1016, Paris, France. ${ }^{7}$ Anatomy and Pathological Cytology Service, GH Cochin-Saint Vincent de Paul, Paris, France and ${ }^{8}$ Plastic Surgery Service, Saint Louis Hospital, Paris, France. Correspondence: M Bomsel (morgane.bomsel@inserm.fr)
} 
cytomegalovirus, adenovirus, papillomavirus, and herpes simplex virus) that commonly infect and may further ascend the male genital tract, ${ }^{11-15}$ demonstrating that the urethral mucosa is accessible to bacterial/viral invasion during sexual intercourse. In addition, early studies in the simian immunodeficiency virus model showed that infusion of cell-free simian immunodeficiency virus into the urethra of male macaques resulted in development of AIDS-like disease, ${ }^{16,17}$ although a more recent study concluded that cell-free simian immunodeficiency virus was inefficiently transmitted to male macaques by penile inoculation. ${ }^{18}$ In men, HIV-1 target cells and receptors are present in the different penile epithelia: ${ }^{9,10,19-21}$ (i) CD1a + cells are present in the glans and fossa, but not in the urethra. Whether these cells are indeed Langerhans cells also expressing the HIV-1-binding C-type lectin langerin ${ }^{22,23}$ is unknown; (ii) $\mathrm{CD} 4+\mathrm{T}$ cells are present in all penile epithelia; (iii) macrophages are observed to some extent in the fossa and seem to be more abundant in the urethra; (iv) cells expressing the HIV-1 co-receptors CCR5 and CXCR4 are present in the glans and urethra. The exact identity and quantitative distribution of these cells in the epithelial and stromal compartments of penile mucosa remain elusive.

In the present study, we aimed at elucidating, for the first time, the paths responsible for HIV-1 penile entry. Whole penile tissues were obtained from individuals undergoing elective gender reassignment, and penile explants were established from the urethra, fossa, and glans. In parallel, a novel in vitro reconstructed urethral mucosa was developed. Using these complementary experimental models, we show herein that macrophages residing within the penile urethra, but not $\mathrm{T}$ cells, are the first cellular targets of HIV-1 on penile exposure.

\section{RESULTS}

\section{Quantitative distribution of potential HIV-1 target cells in the human penis}

To investigate the presence and distribution of penile HIV-1 target cells, sections were prepared from the adult penile urethra, fossa, and glans epithelia, stained with antibodies (Abs) against cell-specific markers (langerin for Langerhans cells, CD3 for T cells, CD68 for macrophages), and examined by immunohistochemistry. The numbers of each cell type were counted in each region, both in the epithelial and stromal compartments, to determine quantitatively and comparatively the respective cell densities (cells per $\mathrm{mm}^{2}$ ). All penile epithelia were also observed by electron microscopy.

The epithelial compartment of the urethra showed a typical pseudostratified columnar morphology with two to three layers of epithelial cells (Figure 1a,e,i,m). The epithelial compartment of the fossa showed a stratified morphology with five to six layers of cells and lacked visible apical keratinization (Figure 1b,f,j,,n). The epithelial compartment of the glans showed a higher degree of stratification, with at least 10 layers of epithelial cells, including terminally differentiated cells at the apical surface forming multiple keratin layers (Figure 1c,g,k,o).
Langerin + cells were completely absent in the epithelial compartment of the urethra (Figure 1a). In contrast, langerin + cells were present in the epithelium of the fossa (Figure 1b) and glans (Figure 1c,p), with a density significantly higher in the glans than in the fossa (Figure 1d). Both the epithelial and stromal compartments of all regions contained $\mathrm{CD} 3+$ cells (Figure 1e-g,r). The epithelial densities of CD3 + cells were comparable, yet their stromal density was significantly higher in the glans compared with the urethra and fossa (Figure 1h). CD68 + macrophages that localized both in the epithelial and stromal compartments in the urethra (Figure 1i,q,r) remained confined to the stromal compartment in the fossa (Figure 1j) and glans (Figure 1k). The stromal densities of CD68 + cells were comparable for all penile regions (Figure 11).

\section{Efficient urethral translocation of cell-associated, but not cell-free, HIV-1 in ex vivo penile explants}

To mimic HIV-1 sexual transmission ex vivo, inoculation of the virus must be restricted to the epithelial/mucosal side of tissue explants. Therefore, tissue pieces derived from the urethra, fossa, and glans were placed with their epithelial side facing up on top of a permeable membrane in a two-chamber system. Hollow plastic cloning ring cylinders were adhered tightly to the epithelial surface of each tissue explant using surgical glue (Figure 2a). This experimental system assures the creation of a sealed apical chamber that permits for polarized inoculation of HIV-1 and was used successfully in our previous studies of foreskin $^{5,7}$ and rectal explants. ${ }^{24}$

In all secretions transmitting HIV-1, the virus is present either as virus-infected cells or as cell-free particles. To compare the relative infectious and entry potential of these sources of HIV-1, penile explants were exposed to either peripheral blood mononuclear cells (PBMCs) infected with the HIV-1 primary isolate 93BR029 (V29; clade B, R5 tropism; maximal apical viral input of 1,000 pg p24, see Methods) or to cell-free HIV-1 V29 (apical viral input of 5,000 pg p24). One hour later, the apical and basal contents were collected and HIV-1 measured in the supernatant fractions by a p 24 enzyme-linked immunosorbent assay (ELISA).

In repeated experiments using penile tissue explants established from different individuals $(n=9)$, the translocation of cell-associated HIV-1 across different penile regions was low and similar to which we previously reported across simple intestinal and cervical epithelia. ${ }^{25}$ Yet, translocation of cellassociated HIV-1 was repetitively and significantly higher across the urethra compared with the fossa and glans, with mean \pm s.e.m. translocation of $1.28 \pm 0.49 \%, 0.06 \pm 0.04 \%$, and $0.06 \pm 0.06 \%$, respectively (Figure $2 \mathbf{b}$, black bars). As explants from all regions were prepared in a similar and comparative fashion for each individual tested, these results demonstrate the occurrence of specific and differential HIV-1 translocation and absence of nonspecific leakage of HIV-1 across the explants. Urethral exposure to PBMCs from HIV-1-positive individuals resulted in comparable degree of translocation (mean \pm s.e.m. of $0.57 \% \pm 0.34, n=3$ ). In contrast, urethral translocation of 

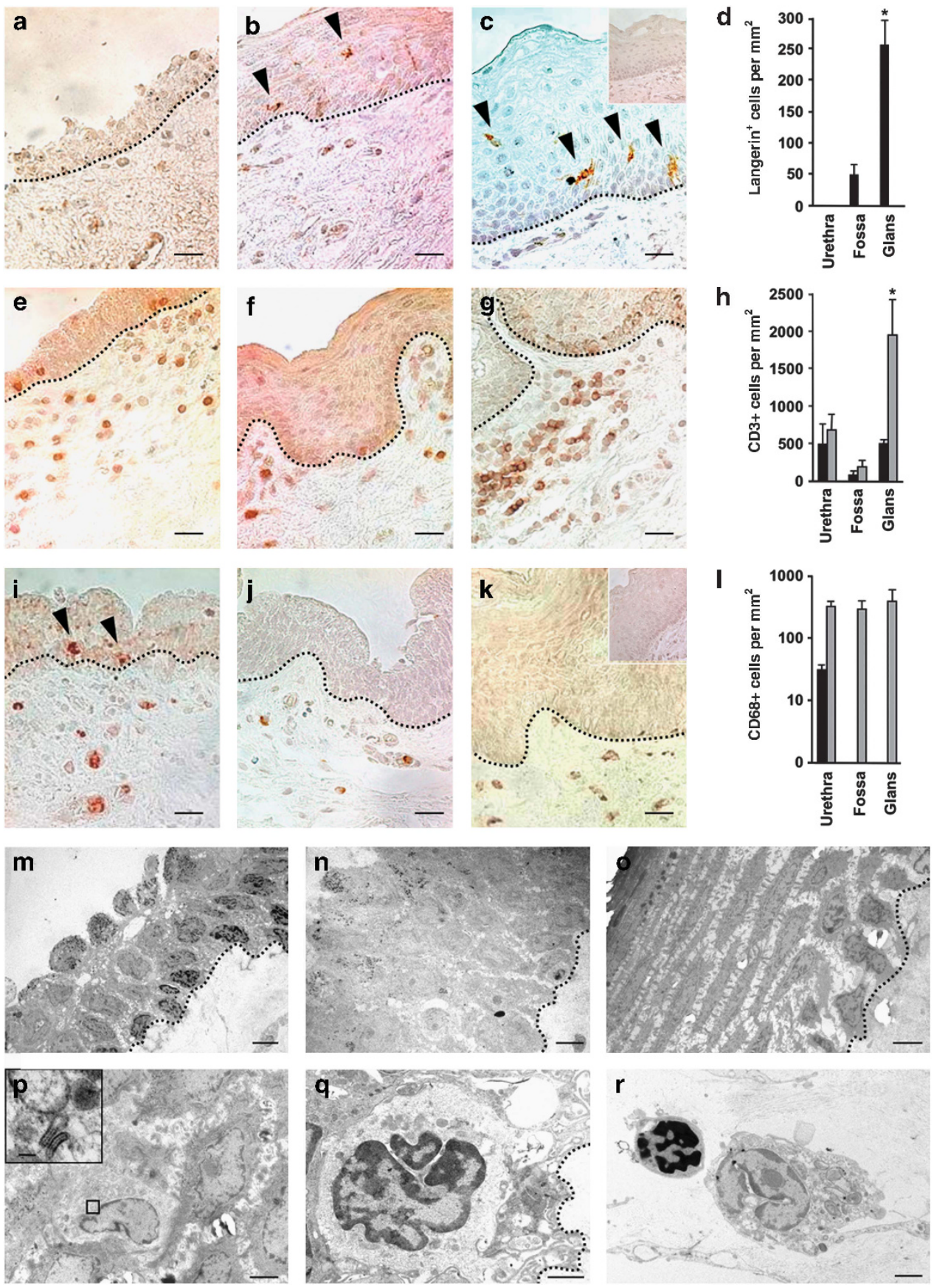

Figure 1 Quantitative distribution of potential HIV type-1 (HIV-1) target cells in different penile epithelia. (a-I) Representative images of the urethra (a, e, i), fossa ( $\mathbf{b}, \mathbf{f}, \mathbf{j})$, and glans $(\mathbf{c}, \mathbf{g}, \mathbf{k})$ processed for immunohistochemistry using antibodies (Abs) to langerin (a-c), CD3 (e- $\mathbf{g})$, and CD68 (i-k), and visualized with either diaminobenzidine (langerin, CD3) or permanent red (CD68) peroxidase substrates. Inserts (c, $\mathbf{k}$ ) show negative staining with matched isotype controls. Black arrowheads denote langerin + (b, c) or CD68 + (i) cells in the epithelial compartments. Bars $=10 \mu \mathrm{m}$. Graphs (d, $\mathbf{h}$, I) show the mean \pm s.e.m. densities of each cells type (cells per $\mathrm{mm}^{2}$ ) for either the epithelial (black bars) or stromal (gray bars) compartments of each region, derived from $n=5$ penile tissues. Statistical analysis: (d) ${ }^{*} P=0.0061$, glans vs. fossa; $(\mathrm{h}){ }^{*} P=0.0338$ and 0.0194 , CD3 in the stromal compartment of the glans vs. urethra and fossa. $(\mathbf{m}-\mathbf{r})$ Representative electron microscopy images from $n=3$ penile tissues: urethra ( $\mathbf{m}$, pseudostratified columnar morphology; q, macrophage in epithelial compartment; $\mathbf{r}$, macrophage (right) and T cell (left) in stromal compartment); fossa (n, stratified morphology with no keratinization); glans (o, highly stratified and keratinized morphology; $\mathbf{p}$, Langerhans cell between epithelial cells (higher magnification insert shows two typical cytoplasmatic rod/racket-shaped Birbeck granules above the cell nucleus)). Bars $=5 \mu \mathrm{m}(\mathbf{m}-\mathbf{p}), 1 \mu \mathrm{m}(\mathbf{q}, \mathbf{r})$, $0.1 \mu \mathrm{m}$ (insert in $\mathbf{p}$ ). Black broken lines in all images denote the basement membranes.

cell-free HIV-1 $(n=3)$ was extremely low and significantly less efficient compared with that of cell-associated HIV-1 (Figure 2b, gray bar; mean \pm s.e.m. of $0.005 \pm 0.005 \%)$. This very low level of cell-free HIV-1 translocation, which is measured following exposure to an even higher apical viral input compared with cell-associated HIV-1 (5,000 vs. 1,000 pg p24, see above), further confirms the lack of nonspecific leakage of viral particles in the explants used herein. 

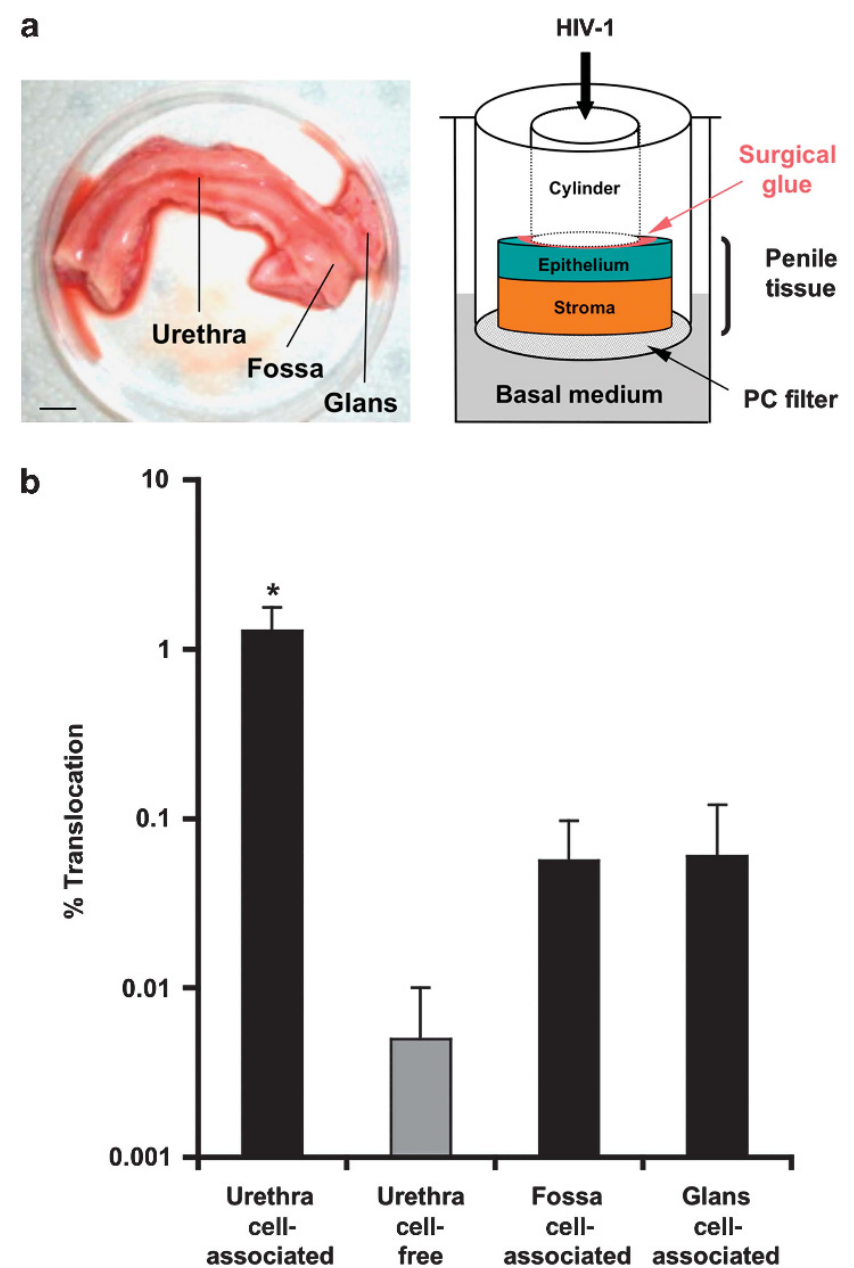

Figure 2 HIV type-1 (HIV-1) translocation across penile explants. (a) A schematic representation of the experimental explant system (right) of different penile epithelia (left). (b) Mean \pm s.e.m. percentages of HIV-1 translocation (p24 in basal chamber/p24 in apical chamber $\times 100$ ) following $1 \mathrm{~h}$ exposure of urethra, fossa, and glans explants to $1 \times 10^{6}$ peripheral blood mononuclear cell (PBMCs) infected with the primary clade B HIV-1 isolate V29 (black bars; maximal apical viral input of $1,000 \mathrm{pg}$ p24) or exposure of urethral explants to cell-free HIV-1 V29 (gray bar; apical viral input of $5,000 \mathrm{pg} \mathrm{p24}$ ). Results are derived from $n=9$ (HIV1-infected PBMCs) and $n=3$ (cell-free HIV-1) explants. Statistical analysis: ${ }^{\star} P<0.0001, P=0.0319$, and $P=0.0329$, urethra cellassociated vs. urethra cell-free, fossa cell-associated, and glans cellassociated, respectively.

Urethral macrophages, but not T cells, are targeted by HIV-1 To identify the initial cells that might be targeted by HIV-1 on its entry into the urethra, urethral explants were exposed in a polarized manner to HIV-1 V29-infected or non-infected PBMCs for $1 \mathrm{~h}$, co-stained for CD68, CD3, cytokeratin 13 (to identify macrophages, T cells, and epithelial cells), and HIV-1 p24, and examined by confocal microscopy.

In urethral explants exposed to non-infected PBMCs, CD68 + macrophages and CD3 $+\mathrm{T}$ cells were detected both in the epithelial and stromal compartments (Figure 3a,c). Following urethral exposure to HIV-1-infected PBMCs, HIV-1 was detected as free virions in the stromal compartment (Figure 3b) or associated with urethral epithelial cells expressing cytokeratin 13 (Figure 3b,e), suggestive of a transcytosis-like mechanism of HIV-1 translocation. In these explants, CD68 + cells were no longer detected within the epithelial compartment, but only within the stroma (Figure 3b). Quantitative analysis confirmed that the density of CD68 + macrophages significantly decreased in the epithelial compartment and increased in the deep parts of the stroma (Figure 3f). Notably, in these explants HIV-1 was also found colocalized with $\mathrm{CD} 68+$ macrophages in the stromal compartment, including virions that were internalized into these cells, and colocalized with CD68 expressed intracellularly (Figure 3b). Surprisingly, HIV-1 was not colocalized with $\mathrm{CD} 3+\mathrm{T}$ cells in any of the explants examined (Figure 3d).

The levels of sixteen cytokines/chemokines secreted to the apical medium, following $1 \mathrm{~h}$ exposure of urethral explants to either non-infected or HIV-1-infected PBMCs, were next measured. At this early time point, the measured levels represent that of stored homeostatic cytokines/chemokines, rather than de novo synthesis. Among the molecules tested, only CCL2/MCP-1 and interleukin (IL)-6 were secreted at very high levels $\left(25,000\right.$ and $12,000 \mathrm{pg} \mathrm{ml}^{-1}$ maximal secretion, respectively; see Methods for a description of the other cytokines/chemokines tested). Notably, in association with the exit of urethral macrophages from the epithelial compartment (Figure 3f), CCL2/MCP-1 epithelial levels decreased following inoculation with $\mathrm{HIV}$-1-infected compared with non-infected PBMCs (Figure 3g).

To further confirm targeting of urethral macrophages by HIV-1, tissue emigrants were collected 3 days following $1 \mathrm{~h}$ polarized exposure of urethral explants to HIV-1 V29-infected or non-infected PBMCs. To identify infected macrophages or T cells, the emigrants were double-stained for surface CD163 or CD3, and intracellular p24 expression, and examined by flow cytometry. In other experiments, emigrants were labeled with a CD163-PE Ab, positively selected using phycoerythrin (PE) magnetic beads, and further incubated with phytohemagglutinin/IL-2-activated reporter CD4 $+\mathrm{T}$ cells. In the urethra, following exposure to HIV-1 V29-infected PBMCs, a distinct proportion of emigrating CD163 + macrophages (Figure 3h, $\mathrm{R} 1$ gate) were p24+ (Figure $3 \mathbf{h}$, top panel). In contrast, the proportion of emigrating CD3 $+\mathrm{T}$ cells (Figure $3 \mathbf{h}, \mathrm{R} 2$ gate) that were p $24+$ was negligible (Figure $3 \mathbf{h}$, bottom panel). The proportions of p $24+$ macrophages and $\mathrm{T}$ cells in the fossa and glans were also similar to background level. When CD163+ macrophages were positively selected from the emigrating cells and incubated with reporter cells, viral replication was evident in the supernatant of CD163 + cells from the urethra, but not from the fossa and glans (Figure 3i). Altogether, these results suggest that urethral macrophages are the initial cells infected by HIV-1 on penile entry.

\section{Expression of HIV-1 receptors and phenotype of penile macrophages and $\mathrm{T}$ cells}

To examine the expression of HIV-1 receptors/co-receptors and phenotype of penile macrophages and T cells, which might be related to their differential permissiveness to HIV-1 (see 
above), single-cell suspensions were prepared from the different penile regions (including both stromal and epithelial compartments for either the urethra or fossa, and separately for the glans, see Methods). The released cells were then co-stained with Abs against CD163 and CD3, as well as CCR5, CD4, CD8, and CD45RO, and analyzed by flow cytometry.

$\mathrm{CD} 163+$ macrophages in all penile regions (Figure 4a, R1 gate) expressed both CD4 and CCR5. Although CD4 expression levels on $\mathrm{CD} 163+$ macrophages from different penile regions were not statistically different, CCR5 expression was significantly higher in the glans compared with the urethra and fossa (Figure 4a). CXCR4 was expressed at comparable levels to CCR5 on $\mathrm{CD} 163+$ urethral macrophages (mean \pm s.e.m. of $16.04 \% \pm 0.85, n=3$ ). CD3 $+\mathrm{T}$ cells in all penile regions (Figure $\mathbf{4 b}, \mathrm{R} 2$ gate) expressed comparable levels of CCR5, CD8, and CD4 (Figure 4b, graph). Yet, CD8 + $\mathrm{T}$ cells significantly outnumbered $\mathrm{CD} 4+\mathrm{T}$ cells in all penile regions, with means \pm s.e.m. of $49.6 \pm 1.8 \% \mathrm{CD} 8+\mathrm{T}$ cells compared with $14.3 \pm 1.1 \% \mathrm{CD} 4+\mathrm{T}$ cells $(P<0.0001)$. A significantly higher proportion of CD8 + were of the memory phenotype, as the $\mathrm{T}$ cell memory marker $\mathrm{CD} 45 \mathrm{RO}$ was expressed on $15.3 \pm 3.3 \% \mathrm{CD} 8+\mathrm{T}$ cells compared with $5.2 \pm 1.2 \%$ on CD $4+\mathrm{T}$ cells $(P=0.0196$; Figure $4 \mathbf{b})$. Further analysis of these memory $\mathrm{T}$ cells revealed that they were of the effector memory phenotype, ${ }^{26,27}$ as they lacked the expression of CCR7 and CD62L (Figure 4b).

\section{Efficient translocation of cell-associated HIV-1 R5, but not $\mathrm{X}$, across in vitro urethral reconstructions is decreased by gp41-specific IgAs}

A major impediment in studying HIV-1 mucosal transmission using penile explants is the small tissue size of each different penile region available for experimental manipulation. Therefore, we established an alternative three-dimensional in vitro model of the urethra (Figure 5a, see Methods for detailed description of the experimental protocol), based on our previous successful reconstruction of an immunocompetent foreskin epithelium. ${ }^{5}$

When compared with normal urethra (Figure 1), urethral reconstructions presented similar morphological characteristics, with a stromal compartment clearly distinct from the overlying two to three apical layers of urethral epithelial cells (Figure 5b). Immunohistochemistry confirmed that CD68 + macrophages were integrated into the urethral reconstructions, into both the epithelial and stromal compartments (Figure 5b).

To compare the urethral permissiveness to HIV-1 strains of different tropism, urethral reconstructions were exposed for $1 \mathrm{~h}$ to PBMCs infected with clade B primary isolates of either R5 (93BR029; V29 isolate) or X4 (91US054; V54 isolate) tropism, as well as to cell-free HIV-1 V29, and HIV-1 translocation was then measured as before. The translocation of cell-associated HIV-1 V29 R5 was dose-dependent and ranged from $5.0 \pm 0.3 \%$ to $7.2 \pm 0.5 \%$ of the apical p24 inputs inoculated (Figure $5 c$, diamonds). The translocation of cell-associated HIV-1 V54 of X4 tropism, although dose-dependent, was significantly less efficient (Figure $\mathbf{5 c}$, circles) and ranged from $2.6 \pm 0.2 \%$ to $4.7 \pm 0.3 \%$ for similar apical p 24 inputs. Cell-free HIV-1 V29 failed to translocate to the basal compartment at low and mid apical p24 inputs, and only $0.6 \pm 0.2 \%$ of the highest p24 apical input translocated after $1 \mathrm{~h}$ (Figure 5c, triangles). As for urethral explants, the limited translocation of cell-free HIV-1 demonstrates the absence of nonspecific leakage of viral particles in the reconstructed urethra. Quantitative evaluation of the densities of $\mathrm{CD}^{+} 8^{+}$ macrophages (cells per $\mathrm{mm}^{2}$ ) in urethral reconstructions exposed to either HIV-1 V29-infected or non-infected PBMCs showed that their epidermal density decreased (from $884 \pm 70$ to $323 \pm 30 ; n=3, P=0.0018$ ) and stromal density increased (from $1,389 \pm 10$ to $1,720 \pm 20 ; n=3, P=0.0415$ ), as observed above for urethral explants (Figure 3f).

The apical poles of additional urethral reconstructions were next pre-incubated with the 2F5 IgA Ab, as our previous studies demonstrated that the monomeric form of this mucosal neutralizing anti-gp41 Ab efficiently inhibits HIV-1 translocation across rectal mucosal tissue explants. ${ }^{24}$ Control reconstructions were pre-incubated with a nonspecific IgA $\mathrm{Ab}$ or culture medium alone. All reconstructions were then exposed for $1 \mathrm{~h}$ to HIV-1 V29-infected PBMCs, and HIV-1 translocation was measured as before. Pre-treatment with the control IgA Ab did not affect the translocation of cell-associated HIV-1 (Figure 5d, gray bar). In contrast, the gp41-specific 2F5 IgA Ab significantly decreased viral translocation in a dose-dependent manner (Figure 5d, red bars).

\section{DISCUSSION}

Our previous studies described the early events of HIV-1 entry into the inner foreskin, ${ }^{5-7}$ rationalizing at the cellular and molecular levels the protective efficacy of circumcision against HIV-1 acquisition. ${ }^{2-4}$ Yet, the foreskin might not necessarily represent the principle/unique penile HIV-1 infection site. The penile urethra is an additional highly probable HIV-1 penile entry site, based on previous observations that various bacterial and viral pathogens that infect the human male genital tract gain access via the urethra, ${ }^{11-14}$ as well as studies in the simian immunodeficiency virus model showing systemic infection following urethral exposure. ${ }^{16,17}$

Structurally, the urethra is a pseudostratified epithelium that is completely different from either tight monolayered (e.g., intestine, rectum) or stratified (e.g., vagina) epithelia, and it is not keratinized alike the foreskin or the glans. In fact, the urethra is the only example of a pseudostratified epithelium among genital/anorectal epithelia, and HIV-1 transmission across such epithelium was never studied before. As shown herein, HIV-1 urethral translocation meets two criteria consistent with a transytosis-like entry mechanism as the one we originally described: ${ }^{25}$ (i) it is rapid; and (ii) it is induced by cell-associated but not cell-free HIV-1. However, despite our morphological evidence for a transcytosis-like mechanism, we cannot exclude a paracellular transport pathway for HIV-1, as the urethral pseudostratified epithelial barrier may not be as tight as monostratified ones. 
Phenotypic analysis of urethral macrophages and $\mathrm{T}$ cells showed that CCR5 is expressed on both cell types. Yet, following $1 \mathrm{~h}$ exposure of urethral explants to cell-associated HIV-1 R5 and viral entry into the urethra, urethral macrophages but not $\mathrm{T}$ cells are the initial cells targeted by HIV-1. We speculate that the early targeting of urethral macrophages is a result of their location within the epithelial compartment, positioning them in close proximity to the apical surface from which HIV-1 enters. In contrast, as the majority of urethral T cells are CD8 + , and as previous studies reported that urethral $\mathrm{CD} 4+\mathrm{T}$ cells are primarily restricted to the stromal compartment, ${ }^{9,11,20} \mathrm{CD} 4+\mathrm{T}$ cells might be less abundant/ accessible to immediate targeting by HIV-1. In addition, only a minority of urethral CD4 + is of the memory subpopulation, which is that preferentially infected/depleted in both the female genital and gastrointestinal tracts. ${ }^{28,29}$

Our findings suggest that urethral macrophages might be important in facilitating urethral HIV-1 transmission. Macrophages contribute to HIV-1 entry also in other mucosal epithelia, as CD4 + /CCR5 + vaginal and ectocervical, but not intestinal, macrophages support HIV-1 R5 entry and replication. Such reduced HIV-1 permissiveness of intestinal macrophages is due to the intestinal cytokine microenvironment that decreases the levels of HIV-1 receptors on these cells, ${ }^{30}$ suggesting that the distinct urethral chemokine/cytokine milieu might affect HIV-1 urethral entry. Specifically, the high levels of the potent monocyte/macrophage chemokine CCL2/MCP-1 might mediate the continuous recruitment of circulating blood monocytes into the urethra, which will then differentiate locally into resident urethral macrophages. Moreover, the decreased secretion of CCL2/MCP-1, reported herein, following urethral exposure to cell-associated HIV-1 might facilitate the exit of urethral macrophages from the epithelial to stromal compartment.

Macrophages colocalized with HIV-1 early on infection of our urethral explants. As macrophages are relatively resistant to HIV-1 infection and are considered a cellular reservoir for HIV $-1,{ }^{31}$ our findings may provide a possible link to the establishment of cellular reservoirs in the male genitals. Several lines of evidence suggest that the internal organs of the male genital tract are anatomical HIV-1 reservoirs, in which macrophages colocalize with HIV- $1{ }^{32}$ Hence, we speculate that the penile urethra might serve as a yet unrecognized anatomical reservoir for HIV-1, which would be potentially sustained in resident macrophages. Our preliminary results indeed show that HIV-1-infected macrophages are detected in the urethral stromal compartment in penile tissues obtained from HIV-1-positive individuals (manuscript in preparation).

We further show that the translocation potential of HIV-1 depends on viral origin and tropism. Hence, cell-associated HIV-1 tranlocates across the urethra much more efficiently compared with cell-free HIV-1, and urethral transmission of R5 compared with X4 strains is more efficient. Similar results were obtained in our previous studies using foreskin explants/ reconstructions, ${ }^{5,6}$ pointing to the crucial contribution of cellassociated HIV-1 to mucosal transmission in the male genitals, and probably other mucosal epithelia, which is overlooked in other studies (for review see ref. 33). Moreover, our results are in agreement with previous observations that mucosal HIV-1 transmission is associated almost exclusively with R5 viruses. ${ }^{34}$

As further demonstrated herein, in "proof-of-concept" experiments using urethral reconstructions, translocation of cell-associated HIV-1 is significantly decreased by gp41specific IgAs. Previous studies have shown that the human urethra harbors components required for the induction of an IgA humoral response. ${ }^{9-11}$ We suggest that mucosal IgAs could potentially inhibit urethral HIV-1 entry if such Abs are directed against the HIV-1 envelope and are induced by an appropriate immunization route (i.e., combination of oral/nasal and intramuscular routes), based on our recent demonstration that immunization of female macaques with gp41 subunit virosomes confers protection from repeated low-dose vaginal challenge with simian/human immunodeficiency virus (SHIV), which is associated with the development of gp41-specific vaginal IgAs with HIV-1 transcytosis-blocking properties. ${ }^{35}$

Taken together, the present study identifies the male adult penile urethra as a novel and unrecognized entry site for HIV-1. The penile urethra may be the primary HIV-1 acquisition site in

Figure 3 Targeting of urethral macrophages by HIV type-1 (HIV-1). (a-e) Representative single sections from $n=3$ different urethral explants observed by confocal microscopy, following $1 \mathrm{~h}$ exposure to $1 \times 10^{6}$ non-infected (a, c) or HIV-1 V29-infected (b, d, e) peripheral blood mononuclear cells (PBMCs). Sections were double-stained with a biotinylated polyclonal antibody (Ab) against p24 followed by Alexa633-conjugated streptavidin, as well as mouse anti-human CD68 (a, b), CD3 (c, d), or cytokeratin 13 (e) Abs followed by Cy3-conjugated anti-mouse IgG Ab. HIV-1 virions are detected as green dots in the stromal compartment (b, single-starred insert), associated with urethral epithelial cells (b, double-starred inserts) expressing also cytokeratin 13 (red, e), or colocalized with CD68 + macrophages (yellow, middle panel in b), but not with CD3 + T cells (red, d). Three-dimensional reconstructions (b, d) were assembled from 35 to 40 single optical sections, taken $0.2 \mu \mathrm{m}$ apart. Cell nuclei were counter-stained with DAPI (4,6-diamidino-2phenylindole; blue). White broken lines in all images denote the basement membranes that separate the epithelial (E) and stromal (S) compartments. Bars $=15 \mu \mathrm{m}(\mathbf{a}, \mathbf{b}$, left column, $\mathbf{c}-\mathbf{e}$ upper panel), $10 \mu \mathrm{m}$ (b, middle column), $5 \mu \mathrm{m}$ (e, lower panel). (f) Mean \pm s.e.m. densities of CD68 + cells (cells per $\mathrm{mm}^{2}$ ) for either the epithelial (black bars) or stromal (gray bars) compartments; ${ }^{*} P=0.0296$ and 0.0392 , V29-infected vs. non-infected PBMCs, respectively, $n=3$. (g) Mean folds \pm s.e.m. epithelial secretion of CCL2/MCP-1 by urethral explants, calculated as (secretion after $1 \mathrm{~h}$ exposure to HIV-1-infected PBMCs/secretion after $1 \mathrm{~h}$ exposure to non-infected PBMCs); ${ }^{*} P=0.0263$, V29-infected vs. non-infected PBMCs, $n=2$. (h) Representative dot plots of urethral emigrants collected 3 days following $1 \mathrm{~h}$ of polarized exposure to $1 \times 10^{6}$ non-infected or HIV-1 V29-infected PBMCs. Left plots show the presence of CD163 + macrophages and CD3 + T cells (R1 and R2 gates vs. isotype control staining, respectively). Right plots show double staining with APC-conjugated Abs to either CD163 or CD3 (surface) and a PE-conjugated Ab to p24 (intracellular). Numbers represent mean \pm s.e.m. proportions of double-positive cells (framed windows) derived from $n=5$ different penile tissues. ${ }^{\star} P<0.0001$, CD163 + p24 + cells following exposure to V29-infected vs. non-infected PBMCs. (i) Penile emigrants were stained with a PE-conjugated CD163 Ab and positively selected using PE magnetic beads. Shown are means \pm s.e.m. HIV-1 replication $\left(\mathrm{pg} \mathrm{ml}{ }^{-1} \mathrm{p} 24\right)$ in supernatants of positively selected CD163 + emigrants from the urethra, fossa, and glans, from $n=3$ experiments, incubated for a week with either medium alone (gray bars) or phytohemagglutinin (PHA)/interleukin (IL)-2-activated reporter CD $4+\mathrm{T}$ cells (black bars). ${ }^{*} P=0.0017$, reporter cells vs. medium in the urethra. 
circumcised men and possibly have a role also in noncircumcised men. Urethral HIV-1 entry should also be accounted for when developing new strategies to limit HIV1 transmission in men and when considering circumcision as a means to reduce HIV-1 transmission.

\section{METHODS}

Tissue. Whole penile tissues were obtained from 24 individuals undergoing elective gender reassignment at the Saint Louis Hospital in Paris, France (mean age 35 years old, range 21-54). Hormonal treatment was ceased 4 weeks before surgery, and all individuals had no history of sexually transmitted infections during the 6 months before a

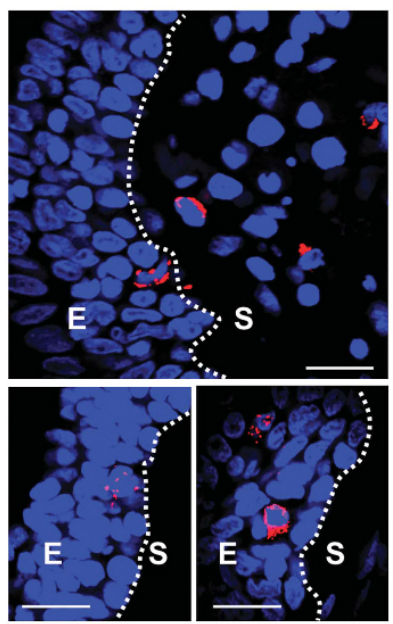

C
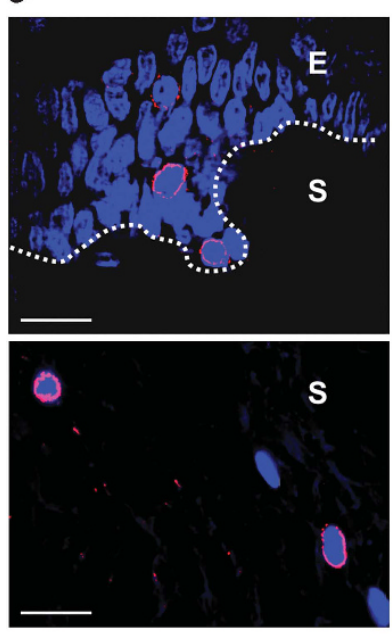

h
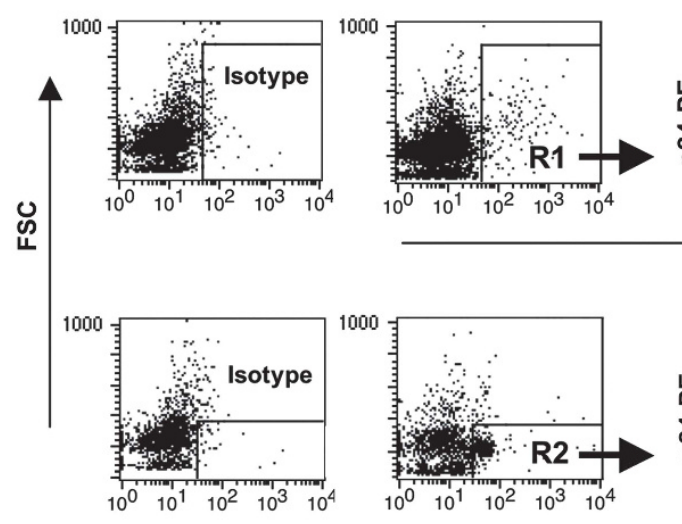

b
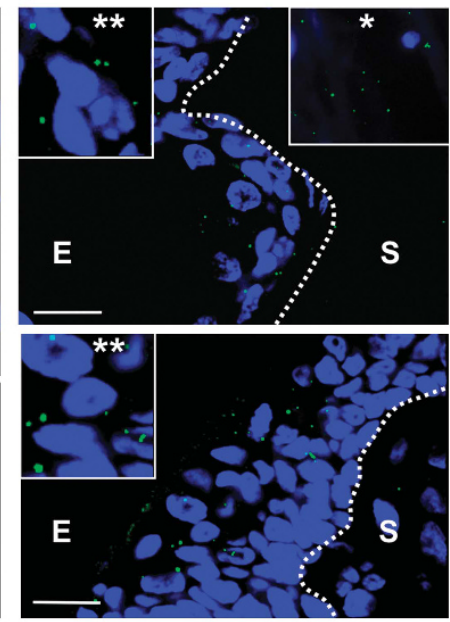

d

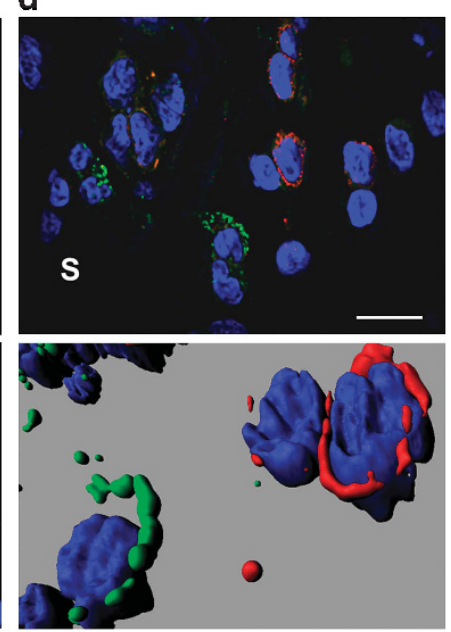

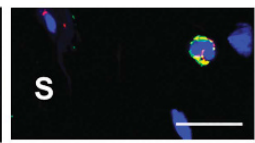
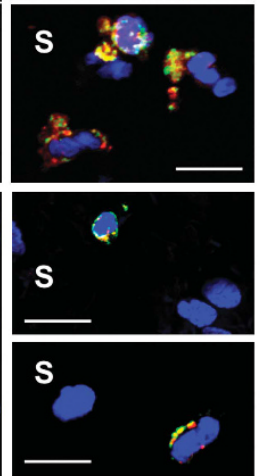

e
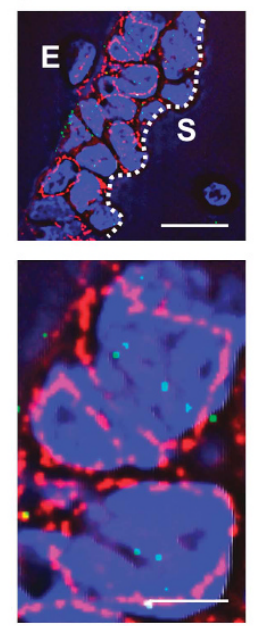

+Non-infected PBMCs
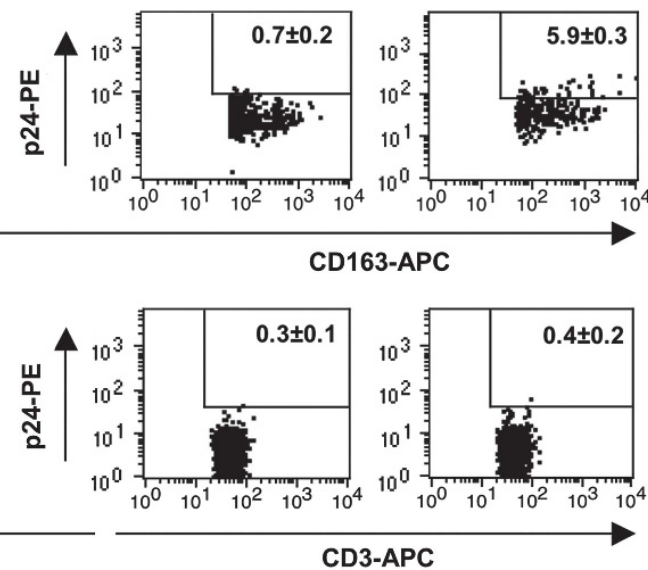
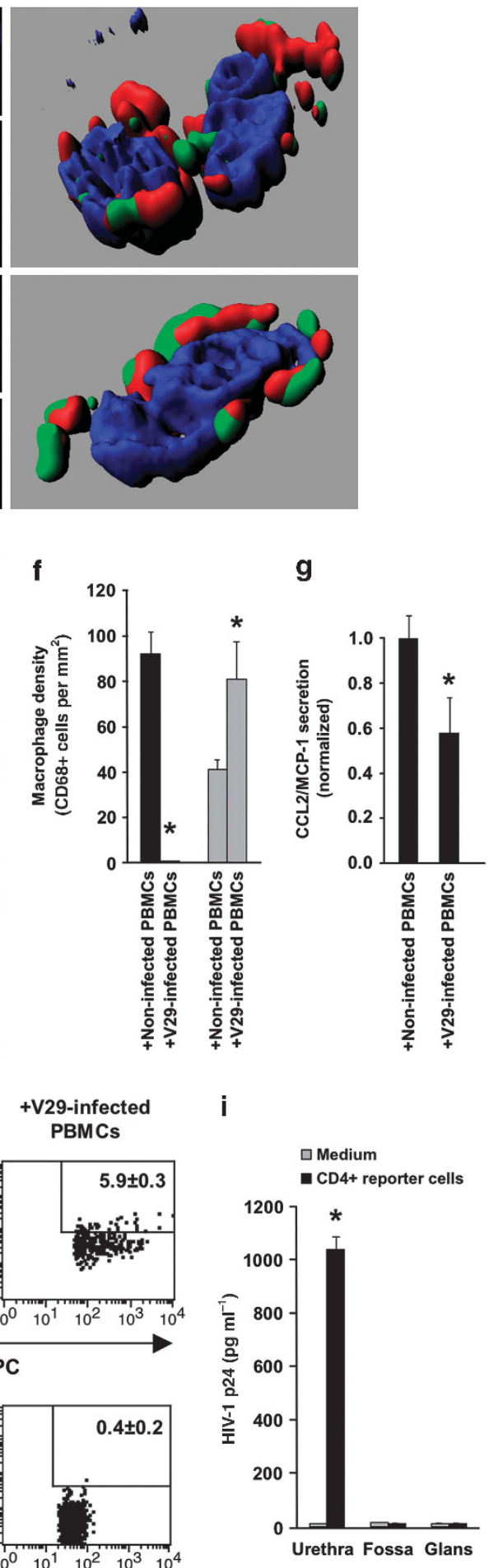

Figure 3 For caption please refer page 781. 


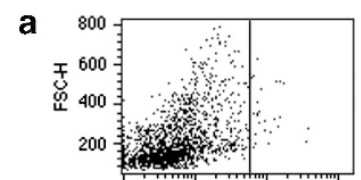

Isotype-APC

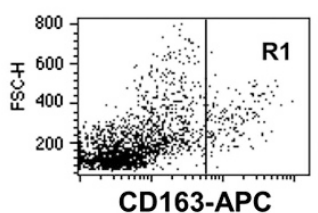

CD163-APC

b

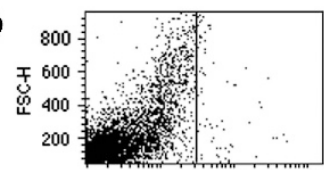

Isotype-APC

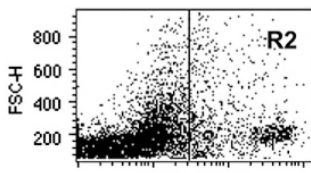

CD3-APC

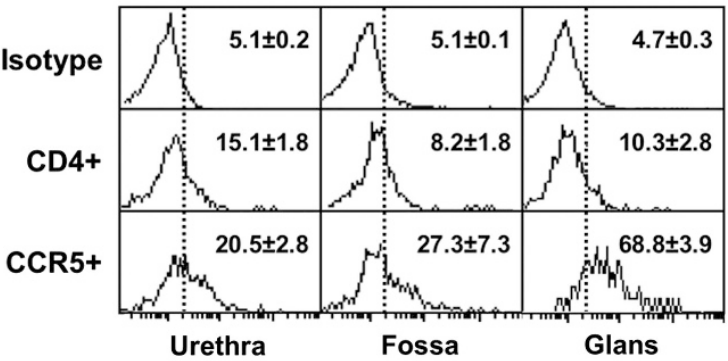

Urethra

Fossa

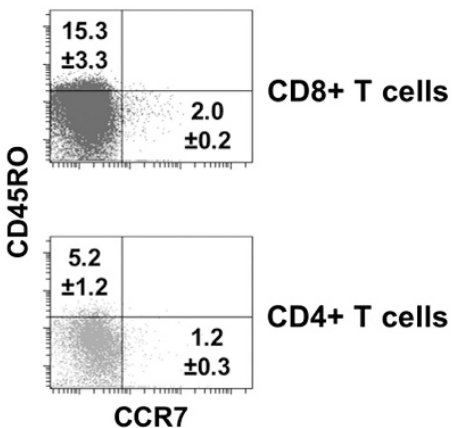

CCR7

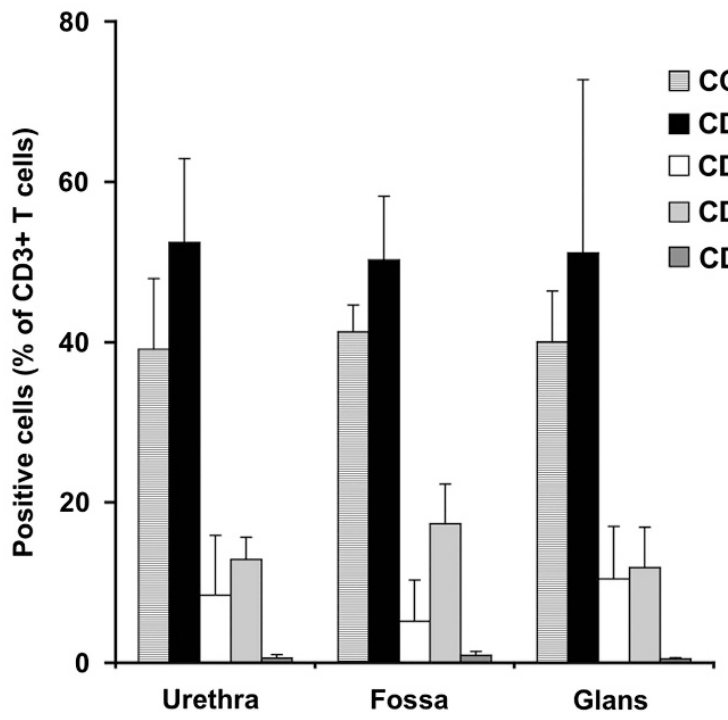

Figure 4 Phenotype of penile macrophages and T cells. Representative dot plots derived from urethral single-cell suspensions showing the presence of CD163 + macrophages (a left, R1 gate) or CD3 + T cells (b left, R2 gate) vs. isotype controls. Representative histograms of R1-gated $\mathrm{CD} 163+$ macrophages (a right, $n=3$ ) derived from all penile regions show cells staining positive for CD 4 or CCR 5 vs. isotype control. Representative plots of R2-gated urethral CD3 + T cells, also expressing either CD8 or CD4 (b right, $n=2$ ), show cells staining positive for either CD45RO or CCR7. Numbers represent the mean \pm s.e.m. proportions of positive cells. The graph in $\mathbf{b}$ shows mean \pm s.e.m. proportions of R2-gated CD3 + T cells derived from all penile regions expressing CCR5 (hatched bars), CD8 (black bars) or CD4 (light gray bars) derived from $n=5$ different penile tissues, as well as the memory T-cell marker CD45RO on CD8 +/CD4 + T cells (white and dark gray bars, respectively) from $n=2$ additional penile tissues.

surgery. Tissues were placed in phosphate-buffered saline supplemented with $20 \mu \mathrm{g} \mathrm{ml}^{-1}$ gentamycin (Gibco Invitrogen, Carlsbad, $\mathrm{CA})$, transported to the laboratory immediately following surgery, and processed within the next $2 \mathrm{~h}$.

Immunohistochemistry, electron, and confocal microscopy. Immunohistochemistry, electron, and confocal microscopy on paraformaldehyde-fixed sections derived from normal urethra, fossa, and glans tissue explants or insert-grown urethral reconstructions were performed as we previously described. ${ }^{5-7}$ Abs used for immunohistochemistry were as described before ${ }^{5-7}$ and staining was visualized with diaminobenzidine (CD3 and langerin staining) and permanent red or 3-amino-9-ethylcarbazole (CD68 staining) peroxidase substrates. Cell quantification was performed using the ImageJ software (NIH) as we previously described. ${ }^{5-7}$ Abs used for confocal microscopy were mouse IgG anti-human CD68, CD3 (DakoCytomation, Glostrup, Denmark) and cytokeratin 10/13 (Abcam, Cambridge, UK) at $10 \mu \mathrm{g} \mathrm{ml}^{-1}$, as well as a biotinylated polyclonal $\mathrm{Ab}$ recognizing HIV-1 p24 (Virostat, Portland, ME) at $20 \mu \mathrm{g} \mathrm{ml}^{-1}$. Secondary Abs were Cy3-conjugated anti-mouse IgG (Jackson Immunoresearch, West Grove, PA) and Alexa633-conjugated 


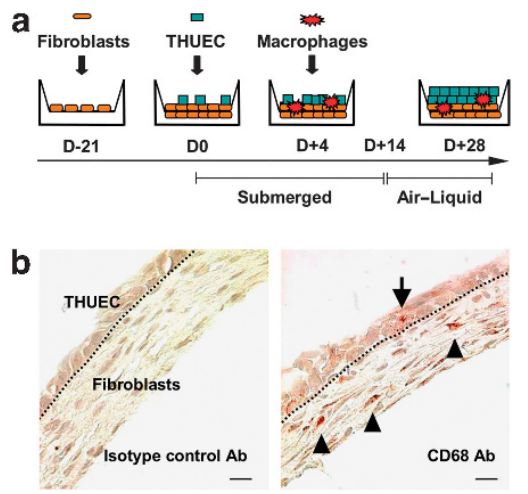

c

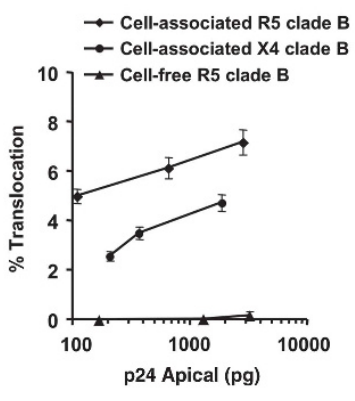

d

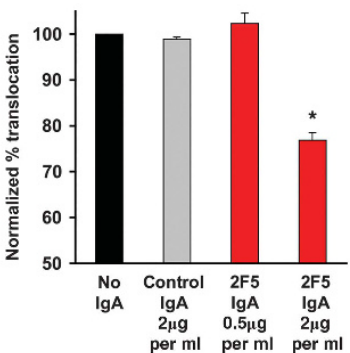

Figure 5 HIV type-1 (HIV-1) translocation across urethral reconstructions. (a) A schematic representation of the experimental protocol used to reconstruct in vitro an immunocompetent urethral mucosa (D, day of culture; THUEC, transformed human urethral epithelial cell line). (b) Immunohistochemistry of reconstructed urethra stained with an antibody $(\mathrm{Ab})$ against CD68 (right; red staining with 3-amino-9-ethylcarbazole peroxidase substrate) or matched isotype control Ab (left). Black arrow and arrowheads denote CD68 + macrophages integrated either within the epithelial or stromal compartments, respectively. Images are representative of $n=4$ urethral reconstructions. Bars $=10 \mu \mathrm{m}$. (c) Mean \pm s.e.m. percentages of HIV-1 translocation (p24 in basal chamber/p24 in apical chamber $\times 100)$ following $1 \mathrm{~h}$ exposure of urethral reconstructions to increasing amounts $(0.5,1$, $2 \times 10^{6}$ ) of peripheral blood mononuclear cells (PBMCs) infected with the primary clade B HIV-1 isolates V29 (R5 tropism, diamonds), V54 (X4 tropism, circles), or to increasing amounts $(50-5,000 \mathrm{pg}$ p24) of cell-free HIV-1 V29 (triangles). Results are derived from $n=4$ reconstructions. $P=0.0031$, 0.0170 , and 0.0282 for cell-associated R5 vs. X4; low, mid, and high p24 apical inputs, respectively. (d) Mean \pm s.e.m. normalized percentage of HIV-1 translocation (\% translocation in the presence of Abs/\% translocation in the presence of medium alone) following 45 min pre-incubation of urethral reconstructions with $2 \mathrm{~F} 5 \mathrm{IgA}$ (red bars), control IgA (gray bar), or medium alone (black bar), and subsequent $1 \mathrm{~h}$ exposure to $2 \times 10^{6} \mathrm{HIV}-1 \mathrm{~V} 29$-infected PBMCs. Results are derived from $n=3$ reconstructions. ${ }^{*} P=0.0062, \mathrm{HIV}-1$ V29-infected PBMCs $+2 \mu \mathrm{g} \mathrm{ml}{ }^{-1} 2 \mathrm{~F} 5 \mathrm{IgA}$ vs. control IgA.

streptavidin (Invitrogen, Cergy Pontoise, France) at 1:100-200 dilution. Images were acquired with a Leica DMI6000 inverse microscope (Leica Microsystems, Wetzlar, Germany), equipped with a confocal light unit (Innova 70C Argon krypton ion gas laser, operating on all laser lines simultaneously by an acoustooptic tunable filter, based on a modified Yokogawa CSU-10 head (Yokogawa Electric, Tokyo, Japan)). Image analysis and three-dimensional reconstructions were performed as before. ${ }^{5-7}$

Single-cell suspensions and flow cytometry. For the glans, the epidermis was first separated from the dermis by dispase digestion. Similar treatment of the other penile epithelia did not yield apparent epithelial sheets/fragments. Hence, single-cell suspensions of the glans dermis and both epithelial/stromal compartments of the fossa and urethra were obtained by collagenase digestion. These different cell suspensions were prepared as we previously described. ${ }^{5-7}$

For immunofluorescent staining, cells were resuspended in phosphate-buffered saline (devoid of $\mathrm{Ca}^{2+} / \mathrm{Mg}^{2+}$ ions) and transferred to a 96-round-bottom-well plate $\left(0.1-0.5 \times 10^{6}\right.$ cells per well). Cells were incubated for $30 \mathrm{~min}$ on ice with $5-10 \mu \mathrm{g} \mathrm{ml}^{-1}(50 \mu \mathrm{l}$ per well) of mouse anti-human Abs as follows: allophycocyanin (APC)CD163, APC-CD3, and FITC-CCR5/CD4/CD8 (BD Pharmingen, San Jose, CA). In other experiments, staining was performed with APCH7-CD3, Pasific-Blue-CD4, and APC-CD45RO Abs (BD Pharmingen. Cells stained with matched isotype control Abs served as negative controls. Fluorescence profiles were recorded using either a FACSCalibur or a FACSCanto-II, and results were analyzed using the CellQuest Pro or FACSDiva software (BD Biosciences, San Jose, CA), respectively.

Ex vivo polarized penile tissue explants. Penile tissues segments derived from the urethra, fossa, and glans were cut into $8 \times 8 \mathrm{~mm}^{2}$ pieces and the stromal muscle/spongy tissue was removed. Explants were established in two-chamber systems using surgical glue as we previously described. ${ }^{5,7}$ Firm attachment of the cylinders was evident by their retention on the epidermal surface, even when the twochamber system inserts were inverted to an upright position. Furthermore, to verify that the cylinder-based apical chambers are indeed completely sealed, $100 \mu \mathrm{l}$ of either RPMI 1640 medium or trypan blue solution (Gibco Invitrogen) was added to the inner space of the cylinders, and the explants incubated for $4 \mathrm{~h}$ at $37^{\circ} \mathrm{C}$. Following incubation, no signs of leakage were detected as evident by the ability to completely recover the total volume of medium initially added and the complete absence of trypan blue traces around the cylinder edges or in the basal chamber.

In vitro urethral reconstruction. Primary human fibroblasts derived from inner foreskin were purified and maintained as we previously described. $^{5}$ The transformed human urethral epithelia cell line THUEC was a kind gift of Professor MA Apicella (University of Iowa, Iowa City, IA). This cell line was characterized previously and maintained as described. ${ }^{36}$ PBMCs from healthy donors were separated from whole blood by a standard Ficoll gradient, and CD14 + monocytes were obtained using a negative magnetic selection kit (Stemcell Technologies, Grenoble, France) according to the manufacturer's instructions. Cells were cultured as before, ${ }^{5,7}$ and macrophages were generated by adding $100 \mathrm{ng} \mathrm{ml}^{-1}$ granular macrophage colony-stimulating factor (Peprotech, Rocky Hill, NJ) for 7 days, as previously described. ${ }^{37}$

Urethral reconstruction was performed according to our recently published protocol, ${ }^{5,7}$ seeding first fibroblasts, and then adding THUEC $\left(2-5 \times 10^{5}\right.$ cells per insert $)$ and macrophages $(0.5-1 \times$ $10^{5}$ cells per insert) 4 days later. Reconstructions were further cultured for 2 weeks under submerged conditions, and then for 2 additional weeks at the air-liquid interface. ${ }^{5,7}$ Of note, $\mathrm{T}$ cells were not incorporated into these urethral reconstructions, as autologous $\mathrm{T}$ cells could not be routinely obtained, and allogeneic $\mathrm{T}$ cells would potentially mediate an immune attack and destruction of the fibroblasts/THUEC used.

Virus and infected cells. The HIV-1 primary isolates 93BR029 and 91US054 (clade B, R5 and X4 tropic, respectively; NIH AIDS reagent program) were amplified on phytohemagglutinin-stimulated PBMCs and quantified by the p24 Innotest HIV-1 ELISA (Innogenetics, Gent, Belgium) as described. ${ }^{38}$ HIV-1-infected cells were obtained as we previously described. ${ }^{5,7}$ Infection was monitored in culture supernatants by p24 ELISA and by p24 intracellular staining and flow cytometry analysis. Infected cells were used between days 7 and 14 following addition of virus. Under these infection conditions, $1 \times 10^{6}$ infected cells, from different donors and at different time points 
following infection, released 100-3,000 pg p24 after $1 \mathrm{~h}$ incubation at $37^{\circ} \mathrm{C}$, and $1-7 \%$ of the infected cells were positive for $\mathrm{p} 24$. The infectious potential of the R5 and X4 isolates used herein was similar.

PBMCs infected with HIV-1 clade B were also purified from blood of HIV-1-positive individuals with elevated plasma viral loads, as described before. ${ }^{39}$ In these preparations, the proportion of HIV-1infected cells ranged from 10,000 to 15,000 infected cells per $5 \times 10^{6}$ PBMCs.

Short-term polarized infection assay. Penile explants or urethral reconstructions were exposed in a polarized manner via the epithelial side to $0.5-2 \times 10^{6}$ of either HIV-1-infected or non-infected PBMCs diluted in RPMI 1640 medium ( $100 \mu$ ladded into the inner space of the cloning ring cylinders adhered to the tissue explants or $200 \mu \mathrm{l}$ added to the upper chamber of urethral reconstructions; lower chambers were supplemented with $0.5 \mathrm{ml}$ RPMI 1640). Where indicated, explants/ reconstructions were also exposed to cell-free virus $(5,000 \mathrm{pg}$ p24 for explants, 50-5,000 pg p24 for reconstructions). In some experiments, urethral reconstructions were first pre-incubated for $45 \mathrm{~min}$ at $37^{\circ} \mathrm{C}$ with 0.5 or $2 \mu \mathrm{g} \mathrm{ml}^{-1}$ of either the 2F5 IgA, we produced and described before, ${ }^{24}$ or a purified nonspecific control IgA (The Binding Site, Birmingham, UK). Following $1 \mathrm{~h}$ incubation at $37^{\circ} \mathrm{C}$, the content of both apical and basal chambers was removed, centrifuged, and the supernatant fractions were aliquoted and stored at $-80^{\circ} \mathrm{C}$ for further quantification of HIV-1 by p24 ELISA.

Tissue emigrants. To collect emigrants following polarized HIV-1 inoculation, penile explants were washed and further incubated at $37^{\circ} \mathrm{C}$ for 3 days in a non-polarized manner in $3 \mathrm{ml}$ RPMI 1640/ explant. Emigrants were collected from the culture medium, stained with APC-CD163 or APC-CD3 Abs (or matched isotype control Abs) as described above, centrifuged, and fixed for $15 \mathrm{~min}$ at room temperature with $4 \%$ paraformaldehyde. Fixed cells were then permeabilized for $15 \mathrm{~min}$ at room temperature with phosphate-buffered saline containing $0.1 \%$ saponin, and then incubated for $30 \mathrm{~min}$ at room temperature with PE-conjugated mouse anti-human Ab to HIV-1 p24 and core antigens (Beckman Coulter, Fullerton, CA), diluted 1:160 in phosphate-buffered saline $/ 0.1 \%$ saponin to a final volume of $50 \mu \mathrm{l}$ per well. Cells were analyzed with a FACSCalibur as described above. In some experiments, emigrants were stained with a PE-CD163 Ab (BD Pharmingen) as above, and the PE-labeled cells selected using the PE Selection Kit and EasyPlate (Stemcell Technologies) according to the manufacturer's instructions. Selection purity was $85-90 \%$ as estimated by flow cytometry analysis of the labeled cells. The positively selected cells were then incubated in a 96-round-bottom-well plate (approximately $0.5 \times 10^{5}$ cells per well per $200 \mu \mathrm{l}$ ) in RPMI 1640 medium supplemented with $10 \%$ fetal bovine serum (PAN Biotech $\mathrm{GmbH}$, Aidenbach, Germany), $2 \mathrm{~mm}$ L-glutamine, $100 \mathrm{U} \mathrm{ml}^{-1}$ penicillin, and $100 \mu \mathrm{g} \mathrm{ml}^{-1}$ streptomycin (Gibco Invitrogen), either alone or in the presence of $0.1 \times 10^{6} \mathrm{CD} 4+\mathrm{T}$ cells (purified from PBMCs using a negative selection kit according to the manufacturer's instructions (Stemcell Technologies), and activated with phytohemagglutinin/IL-2 for 4 days, as described ${ }^{38}$ ). A week later, HIV-1 replication in the supernatant fractions was quantified by $\mathrm{p} 24$ ELISA.

Quantification of secreted cytokines and chemokines. The levels of IL-1 $\beta$, IL-2, IL-4, IL-5, IL-6, IL-9, IL-10, IL-12p70, IL-13, IL-17A, IL22 , interferon- $\gamma$, tumor necrosis factor- $\alpha$, CCL2/MCP-1, CCL3/MIP$1 \alpha$, CCL5/RANTES were measured by a multiplex bead immunoassay kit (Bender MedSystems, Vienna, Austria) and quantified by flow cytometry, according to the manufacturer's instructions.

Statistical analysis. Statistical significance was analyzed by two-tailed Student's $t$-test.

Ethical statement. The study was performed according to local ethical regulations, following approval by the local ethical committee (Comité de Protection des Personnes, Île de France XI, approval number 11 016). All study participants provided written informed consents.

\section{ACKNOWLEDGEMENTS}

We thank M.A. Apicella (The University of lowa, lowa, USA) for his kind gift of the THUEC cell line, and C. Lesaffre and M. Favier (Cochin Institute's Morphology and Histology platform). Y.G. was supported by Agence National de Recherche sur le SIDA et les Hépatites (ANRS) fellowship, and Z.Z. was supported by the SIDACTION fellowship. M.B. was supported by grants from ANRS.

c 2013 Society for Mucosal Immunology

\section{REFERENCES}

1. Hladik, F. \& McElrath, M.J. Setting the stage: host invasion by HIV. Nat ReV Immunol 8, 447-457 (2008).

2. Auvert, B. et al. Randomized, controlled intervention trial of male circumcision for reduction of HIV infection risk: the ANRS 1265 Trial. PLoS Med 2, e298 (2005).

3. Bailey, R.C. et al. Male circumcision for HIV prevention in young men in Kisumu, Kenya: a randomised controlled trial. Lancet 369, 643-656 (2007).

4. Gray, R.H. et al. Male circumcision for HIV prevention in men in Rakai, Uganda: a randomised trial. Lancet 369, 657-666 (2007).

5. Ganor, Y. et al. Within $1 \mathrm{~h}, \mathrm{HIV}-1$ uses viral synapses to enter efficiently the inner, but not outer, foreskin mucosa and engages Langerhans-T cell conjugates. Mucosal Immunol 3, 506-522 (2010).

6. Ganor, Y. \& Bomsel, M. HIV-1 transmission in the male genital tract. Am J Reprod Immunol 65, 284-291 (2011).

7. Zhou, Z. et al. HIV-1 efficient entry in inner foreskin is mediated by elevated CCL5/RANTES that recruits T cells and fuels conjugate formation with langerhans cells. PLoS Pathog 7, e1002100 (2011).

8. Holstein, A.F., Davidoff, M.S., Breucker, H., Countouris, N. \& Orlandini, G. Different epithelia in the distal human male urethra. Cell Tissue Res 264, 23-32 (1991).

9. Pudney, J. \& Anderson, D.J. Immunobiology of the human penile urethra. Am J Pathol 147, 155-165 (1995).

10. Anderson, D., Politch, J.A. \& Pudney, J. HIV infection and immune defense of the penis. Am J Reprod Immunol 65, 220-229 (2011).

11. Pudney, J. \& Anderson, D. Innate and acquired immunity in the human penile urethra. J Reprod Immuno/ 88, 219-227 (2011).

12. Shigehara, K. et al. Prevalence of human papillomavirus infection in the urinary tract of men with urethritis. Int J Urol 17, 563-568 (2010).

13. Srugo, I. et al. Agents of non-gonococcal urethritis in males attending an Israeli clinic for sexually transmitted diseases. Isr Med Assoc J 5, 24-27 (2003).

14. Bradshaw, C.S. et al. Etiologies of nongonococcal urethritis: bacteria, viruses, and the association with orogenital exposure. J Infect Dis 193, 336-345 (2006).

15. Wagenlehner, F.M., Weidner, W. \& Naber, K.G. Chlamydial infections in urology. World J Urol 24, 4-12 (2006).

16. Ishizaka, S.T. et al. Induction of mucosal antibody responses by microsphere-encapsulated formalin-inactivated simian immunodeficiency virus in a male urethral challenge model. Vaccine 17, 2817-2825 (1999).

17. Miller, C.J. et al. Genital mucosal transmission of simian immunodeficiency virus: animal model for heterosexual transmission of human immunodeficiency virus. J Virol 63, 4277-4284 (1989).

18. Ma, Z.M. et al. SIVmac251 is inefficiently transmitted to rhesus macaques by penile inoculation with a single SIVenv variant found in ramp-up phase plasma. AIDS Res Hum Retroviruses 27, 1259-1269 (2011).

19. McCoombe, S.G. \& Short, R.V. Potential HIV-1 target cells in the human penis. Aids 20, 1491-1495 (2006).

20. Fischetti, L., Barry, S.M., Hope, T.J. \& Shattock, R.J. HIV-1 infection of human penile explant tissue and protection by candidate microbicides. AIDS 23, 319-328 (2009).

21. McClure, C.P. et al. HIV coreceptor and chemokine ligand gene expression in the male urethra and female cervix. AIDS 19, 1257-1265 (2005).

22. Turville, S.G. et al. Diversity of receptors binding HIV on dendritic cell subsets. Nat Immunol 3, 975-983 (2002). 
23. Valladeau, J. et al. Langerin, a novel C-type lectin specific to Langerhans cells, is an endocytic receptor that induces the formation of Birbeck granules. Immunity 12, 71-81 (2000).

24. Shen, R. et al. GP41-specific antibody blocks cell-free HIV type 1 transcytosis through human rectal mucosa and model colonic epithelium. J Immunol 184, 3648-3655 (2010).

25. Bomsel, M. Transcytosis of infectious human immunodeficiency virus across a tight human epithelial cell line barrier. Nat Med 3, $42-47$ (1997).

26. Sallusto, F., Geginat, J. \& Lanzavecchia, A. Central memory and effector memory Tcell subsets: function, generation, and maintenance. Annu Rev Immunol 22, 745-763 (2004).

27. Sheridan, B.S. \& Lefrancois, L. Regional and mucosal memory T cells. Nat Immunol 12, 485-491 (2011).

28. Gupta, P. et al. Memory CD4(+) T cells are the earliest detectable human immunodeficiency virus type 1 (HIV-1)-infected cells in the female genital mucosal tissue during HIV-1 transmission in an organ culture system. J Virol 76, 9868-9876 (2002).

29. Li, Q. et al. Peak SIV replication in resting memory CD4 + Tcells depletes gut lamina propria CD4 + T cells. Nature 434, 1148-1152 (2005).

30. Shen, R. et al. Stromal down-regulation of macrophage CD4/CCR5 expression and NF-kappaB activation mediates HIV-1 non-permissiveness in intestinal macrophages. PLoS Pathog 7, e10020602011).

31. Coiras, M., Lopez-Huertas, M.R., Perez-Olmeda, M. \& Alcami, J. Understanding HIV-1 latency provides clues for the eradication of longterm reservoirs. Nat Rev Microbiol 7, 798-812 (2009).
32. Le Tortorec, A. \& Dejucq-Rainsford, N. HIV infection of the male genital tract-consequences for sexual transmission and reproduction. Int $J$ Androl 33, e98-e108 (2010).

33. Anderson, D.J. et al. Targeting Trojan Horse leukocytes for HIV prevention. Aids 24, 163-187 (2010).

34. Keele, B.F. et al. Identification and characterization of transmitted and early founder virus envelopes in primary HIV-1 infection. Proc Nat/ Acad Sci USA 105, 7552-7557 (2008).

35. Bomsel, M. et al. Immunization with HIV-1 gp41 subunit virosomes induces mucosal antibodies protecting nonhuman primates against vaginal SHIV challenges. Immunity 34, 269-280 (2011).

36. Harvey, H.A., Post, D.M. \& Apicella, M.A. Immortalization of human urethral epithelial cells: a model for the study of the pathogenesis of and the inflammatory cytokine response to Neisseria gonorrhoeae infection. Infect Immun 70, 5808-5815 (2002).

37. Holl, V. et al. Involvement of Fc gamma RI (CD64) in the mechanism of HIV-1 inhibition by polyclonal IgG purified from infected patients in cultured monocyte-derived macrophages. J Immunol 173, 6274-6283 (2004).

38. Lagaye, S. et al. Cell-to-cell contact results in a selective translocation of maternal human immunodeficiency virus type 1 quasispecies across a trophoblastic barrier by both transcytosis and infection. J Virol 75, 47804791 (2001).

39. Bagnarelli, P. et al. Dynamics and modulation of human immunodeficiency virus type 1 transcripts in vitro and in vivo. J Virol 70, 7603-7613 (1996). 